\title{
Whole-exome sequencing identifies a novel mutation in GATA4 gene to be congenital heart defects and arrhythmia in a Chinese family
}

Tianli Zhao

the second xiangya hospital

Guowen Sun

chenzhou First People's Hospital

Yun Zhu

chenzhou no.1 hospital

Yifeng Yang

the second xiangya hospital

Zhiping Tan

the second xiangya hospital

Haisong Bu

the second xiangya hospital

Shijun Hu ( $\nabla$ hushijun@csu.edu.cn )

the second xiangya hospital https://orcid.org/0000-0003-1066-8154

Research article

Keywords: congenital heart disease; GATA4 gene; family tachyarrhythmia

Posted Date: February 21st, 2020

DOl: https://doi.org/10.21203/rs.2.24189/v1

License: @ (i) This work is licensed under a Creative Commons Attribution 4.0 International License. Read Full License 


\section{Abstract}

Background: CHD which contains congenital heart or great vessel structural defects is one of the major birth defects in the world. GATA4 is one of the most widely investigated genes in CHD, with over 100 known mutation sites. Most mutations in the GATA4 gene are related to the structural heart defect.

Methods: 5 patients in this family were enrolled in this study. Punctilious records for the subjects included a complete physical examination, a transthoracic echocardiography, a 12-lead electrocardiograph and a surgical confirming. All noninvasive exam results are confirmed in the surgery. The genomic DNA was extracted from peripheral blood of the patients and their parents. Whole-exome capture and HTS were performed on the proband III.1 by the Novogene Bioinformatics Institute (Beijing, China). Sanger sequencing in forward and reverse directions was used to validate the candidate variants identified by WES, and segregation analyses were performed in the family members.

Results: 3 patients were identified, the father and two of the daughters, in a Chinese family. The daughter proband III.1 was diagnosed as secundum ASD with atrial septal aneurysm, PAVSD, PS and persistent left superior vena cava by transthoracic echocardiography and sinus tachycardia, frequent junctional premature beat with aberrant ventricular conduction. The father II.2 was diagnosed as secundum ASD, PS and ascending aortic dilatation by transthoracic echocardiography and atrial fibrillation paroxysmal ventricular tachycardia. The other daughter III.3 was diagnosed as secundum ASD, mild PS and persistent left superior vena cava by transthoracic echocardiography and sinus arrhythmia (sinus tachycardia alternating with sinus bradycardia), cardiac rhythm migration between atrionector and junction. 1328 variants were detected in the proband's exome. 11 variants were identified and prioritized them by patients' phenotype. The mutation GATA4 NM_002052: exon5: c.T929C: p.M310T could be confirmed in the other two affected family members (II.2 III.3) and could not be detected in other healthy parent or normal control.

Conclusions: In this study, we identified a variant in GATA4 gene (c.T929C: p.M310T) in a CHD family with arrhythmias. It suggested that the region coding NLS of GATA4 gene is an important region for development of heart and GATA4 gene may related to tachyarrhythmias.

\section{Background}

CHD which contains congenital heart or great vessel structural defects is one of the major birth defects in the world. The incidence of $\mathrm{CHD}$ is estimated at approximately $0.89 \%$ in the $\mathrm{China}^{2}$. As the great progress of operation therapy, more CHD patients survive but still be associated with increased risk of cardiovascular disease including stroke ${ }^{3}$, heart failure ${ }^{4}$, and coronary artery heart disease ${ }^{5}$ in later life. CHD is still a serious threat to human, so the early prenatal screening and diagnosis for this type of birth defect are urgently required.

Although the CHD etiology is too complicated to be well characterized because of the complexity of heart development, numerous intrinsic factors such as inherit disease ${ }^{6}$ and extrinsic threats such as environment pollution ${ }^{7}$ were identified contribution to $\mathrm{CHD}$. Genetic factors have been identified as major contribution to the pathogenesis of $\mathrm{CHD}$ with development of sequencing technology in the past two decades ${ }^{8}$. Many TFs 
such as GATA4, TBX5, NKX2.5, BMP4 and HAND1 have been proven to be responsible for heart development and diseases ${ }^{9}$. GATA4 is one of the most widely investigated genes in CHD, with over 100 known mutation sites $^{1}$. Most mutations in the GATA4 gene are related to the structural heart defect such as ASD, VSD, AVSD, DORV, PS and dextrocardia ${ }^{10}$.

In present study, we checked out a clinically characterized family with a diagnosis of CHD. We found an obvious autosomal-dominant inheritance with reduced penetrance $(K=50 \%)$ in this family. We identified a novel mutation GATA4 NM_002052: exon5: c.T929C: p.M310T, by WES of the patient in the family and this mutation was confirmed by sanger sequencing.

\section{Methods}

Patients and clinical examination

5 patients in this family were enrolled in this study (Table 1). Punctilious records for the subjects included a complete physical examination, a transthoracic echocardiography, a 12-lead electrocardiograph and a surgical confirming. All noninvasive exam results are confirmed in the surgery. 
Table 1

Clinical features in members of family

\begin{tabular}{|c|c|c|c|c|c|c|c|c|c|}
\hline \multirow[t]{2}{*}{ Member } & \multirow[t]{2}{*}{ Gender } & \multirow{2}{*}{$\begin{array}{l}\text { Age } \\
\text { (year) }\end{array}$} & \multirow{2}{*}{$\begin{array}{l}\text { Structural } \\
\text { diagnosis }\end{array}$} & \multirow{2}{*}{$\begin{array}{l}\text { ECG } \\
\text { diagnosis }\end{array}$} & \multicolumn{4}{|c|}{ TTE feature } & \multirow[t]{2}{*}{ Mutation } \\
\hline & & & & & $\begin{array}{l}\text { RA } \\
(\mathrm{mm})\end{array}$ & $\begin{array}{l}\text { RV } \\
(\mathrm{mm})\end{array}$ & $\begin{array}{l}\text { LVEF } \\
(\%)\end{array}$ & $\begin{array}{l}\mathrm{AA} \\
(\mathrm{mm})\end{array}$ & \\
\hline$\nabla 1^{a, b}$ & female & 14 & $\begin{array}{l}\text { secundum } \\
\text { ASD with } \\
\text { ASA, } \\
\text { PAVSD, } \\
\text { PS, and } \\
\text { PLSVA }\end{array}$ & $\begin{array}{l}\text { sinus } \\
\text { tachycardia, } \\
\text { frequent } \\
\text { junctional } \\
\text { premature } \\
\text { beat with } \\
\text { aberrant } \\
\text { ventricular } \\
\text { conduction }\end{array}$ & 50 & 37 & 60 & 24 & $\begin{array}{l}\text { WT/p. } \\
\text { M310T }\end{array}$ \\
\hline$凶 2$ & female & 8 & N/A & IRBBB & 22 & 33 & 69 & 17 & WT/ WT \\
\hline$\llbracket 3$ & female & 4 & $\begin{array}{l}\text { secundum } \\
\text { ASD, mild } \\
\text { PS and } \\
\text { PLSVA }\end{array}$ & $\begin{array}{l}\text { sinus } \\
\text { arrhythmia } \\
\text { (sinus } \\
\text { tachycardia } \\
\text { alternating } \\
\text { with sinus } \\
\text { bradycardia), } \\
\text { cardiac } \\
\text { rhythm } \\
\text { migration } \\
\text { between } \\
\text { atrionector } \\
\text { and junction }\end{array}$ & 34 & 31 & 56 & 15 & $\begin{array}{l}\text { WT/p. } \\
\text { M310T }\end{array}$ \\
\hline$\otimes 1$ & male & 48 & $\mathrm{~N} / \mathrm{A}$ & $\mathrm{N} / \mathrm{A}$ & 43 & 40 & 67 & 25 & WT/ WT \\
\hline$\varangle 2$ & male & 44 & $\begin{array}{l}\text { secundum } \\
\text { ASD, PS } \\
\text { and AAD }\end{array}$ & $\begin{array}{l}\text { atrial } \\
\text { fibrillation } \\
\text { paroxysmal } \\
\text { ventricular } \\
\text { tachycardia }\end{array}$ & 71 & 37 & 59 & 37 & $\begin{array}{l}\text { WT/p. } \\
\text { M310T }\end{array}$ \\
\hline$\varangle 3$ & female & 39 & $\mathrm{~N} / \mathrm{A}$ & $\mathrm{N} / \mathrm{A}$ & 40 & 38 & 62 & 22 & WT/ WT \\
\hline
\end{tabular}

The study protocol was approved by Review Board of the Second Xiangya Hospital of Central South University (Changsha, China). Written informed consents for the publication of the patient's information were obtained from the parents of the patient and patents themselves.

DNA extraction

The genomic DNA was extracted from peripheral blood of the patients and their parents. Genomic DNA was prepared using a DNeasy Blood \& Tissue kit (Qiagen, Inc., Valencia, CA, USA) on the QIA cube automated DNA extraction robot (Qiagen, GmbH, Hilden, Germany).

WES and filtering 
Whole-exome capture and HTS were performed on the proband III.1 by the Novogene Bioinformatics Institute (Beijing, China). Briefly, whole exomes were captured by the Agilent SureSelect Human All ExonV5 Kit (Agilent, California, USA) and sequenced on the Illumina HiSEq. 2500 platform. The sequencing reads were aligned to the human reference genome (UCSC hg19), and the details of the sequencing data are provided in Fig. 3. Single nucleotide variants SNVs and short InDels were filtered as follows: (i) Variants within intergenic, intronic, and UTR regions and synonymous mutations were excluded from subsequent analyses. (ii) Highfrequency (minor allele frequency >0.01) polymorphisms found in the 1000 Genomes Project, ESP6500, Exac, and Novogene Bioinformatics Institute in-house exomeSeq databases were excluded. (iii) Based on the principle that the identification of a new disease-causing gene should exclude known causal genes, variants in the 42 CHD-related genes ${ }^{11}$ (Table S1) were selected. This approach prevented the omission of any compound heterozygotes of known genes. (iv) Due to the examination of consanguineous families, runs of homozygosity $(\mathrm{ROH})^{12}$ analysis was performed. $\mathrm{ROH}$ analysis is an important approach because it can effectively exclude false-positive variants in the case of a large deletion on the other allele. The detailed steps of filtering are shown in Fig. 3.

Bioinformatic analysis

The effect of the identified variants on protein function was predicted using bioinformatic programs (Sift, Polyphen-2, MutationTaster, PROVEAN, LRT and MutationAssessor), and potential effects on protein structure were determined using the Swiss Model tool. To evaluate the degree of conservation, alignment of the amino acid sequences of the targeted genes from various species was obtained from MutationTaster (http://www.mutationtaster.org), and domain analyses were performed (http://www.ncbi.nlm.nih.gov/Structure/cdd/wrpsb.cgi).

Mutation validation and co-segregation analysis

Sanger sequencing in forward and reverse directions was used to validate the candidate variants identified by WES, and segregation analyses were performed in the family members. Primer pairs were designed using an online tool (PrimerQuest, IDT), and the sequences of the primers are shown in Table 2.

Table 2

Sequences of the primers used to confirm the mutation by Sanger sequencing

\begin{tabular}{|llll|}
\hline Patient ID & Gene & Variant & Primers \\
\hline ASD family & GATA4 & c.T929C (p.M310T) & F 5'-TCTTTCTCGCTGAGTTCC-3' \\
& & & R 5'-TTGAGTTGAGCCTGCTTC-3' \\
\hline
\end{tabular}

\section{Results}

Clinical features

3 patients were identified, the father and two of the daughters, in a Chinese family (Fig. 1). The daughter proband III.1 was diagnosed as secundum ASD with atrial septal aneurysm, PAVSD, PS and persistent left superior vena cava by transthoracic echocardiography and sinus tachycardia, frequent junctional premature 
beat with aberrant ventricular conduction by HOLTER. The father II.2 was diagnosed as secundum ASD, PS and ascending aortic dilatation by transthoracic echocardiography and atrial fibrillation paroxysmal ventricular tachycardia by HOLTER. The other daughter III.3 was diagnosed as secundum ASD, mild PS and persistent left superior vena cava by transthoracic echocardiography and sinus arrhythmia (sinus tachycardia alternating with sinus bradycardia), cardiac rhythm migration between atrionector and junction by HOLTER. Other members in the family did not show any structural heart defect or arrhythmia in the examination. All structural defects were confirmed in the operation. Proband III.1 presented ventricular tachycardia after sternotomy and the heart was irritable in the whole procedure. All clinical details have been reported in Table1.

Genetic analysis

WES was performed on proband III. 1 and the sequence read of 4.7Gbp was generated. The mean depth of target region is $47.3 \times$ and $95.7 \%$ of the targeted bases was covered sufficiently to pass the threshold for SNVs and InDels (Table S2).

Several filtering methods were performed to analyze the known SNVs and InDels. After alignment and SNV calling, 51328 variants were detected in the proband's exome. We used several databases to exclude all exonic InDels, non-synonymous variants, and nonsense and splice-site SNVs. Then we ranked genes by Sift, Polyphen-2, and MutationTaster, 11 variants were identified and prioritized them by patients' phenotype. 42 CHD-related genes (Table S1) were used to cross-contrast the 11 variants (Fig. 3).

Eventually, only the mutation GATA4 NM_002052: exon5: c.T929C: p.M310T could be confirmed in the other two affected family members (II.2 III.3) and could not be detected in other healthy parent or normal control. M310T mutation was confirmed by Sange sequencing from the samples of available members of the affected family (Fig. 2).

At the same time, several bioinformatic programs (Sift, Polyphen-2, MutationTaster, PROVEAN, LRT and MutationAssessor) were used to confirm the pathogenicity of mutation of M310T (Table 3). The potential effects on protein structure were determined using the Swiss Model tool.

Table 3

Several online databases that used to confirm the pathogenicity of the M310T mutation in GATA4 gene

\begin{tabular}{|llllll|}
\hline Sift & Polyphen-2 & MutationTaster & PROVEAN & LRT & MutationAssessor \\
\hline Damaging & Disease- causing & Disease- causing & Deleterious & Deleterious & Medium \\
\hline
\end{tabular}

\section{Discussion}

Thanks to the development of technique, including introduction of cardiopulmonary bypass and accompanying large-scale heart surgery skills improvement, the morbidity and mortality of CHD have dramatically reduced ${ }^{13}$. However, due to the complexity of heart development, the exact causes of CHD, especially for the different subtype of $\mathrm{CHD}$, still need more ongoing research efforts though we know that knowledge of heart development and function are the absolute necessity for better survival of human. 
Nowadays, the discovery of genetic causes of CHD have been being accelerated by many new technologies including single nucleotide polymorphism arrays, NGS, and copy number variant platforms ${ }^{14}$.

Among all new genetic technologies, the application of NGS in various aspects of heart biology has resulted in new discoveries, generating novel insights into this field of study ${ }^{15}$. In present study, WES combined ROH was employed to find out the suspicious disease-causing gene in one consanguineous family. This technique has been considered as an rapid and cost-effective tool for screening the new variants or genes for rare Mendelian unknown disorders $^{16,17}$. It helps genetic diagnostics for clinical cases with mutational spectrum of known and unknown diseases. Some filtering strategies are needed for excluding variants that are implausible to cause disease because sometimes it is difficult to identify between pathogenic and benign mutations in the WES results ${ }^{18}$. With the analysis of WES result in this study, we quickly determined the most possible pathogenic mutation in this family is GATA4 p.M310T and confirmed the result by sanger sequencing.

CHD families mostly present the same subtype structural defects without arrhythmia. In this study, all affected family members presented similar defects with distinct difference and all 3 patients were detected different tachyarrhythmia. Although some reported arrhythmias and conduction disorders may be associated with atrial septal defects ${ }^{19}$, the reason is unknown and some transcriptional factors genes mutations were related to the ASD family with arrhythmias ${ }^{20}$. To our knowledge, GATA4 has always been regarded as an important role structural development in heart and never been related to tachyarrhythmia. Further investigation is needed for the different phenotype with same genotype in the CHD family with arrhythmias.

GATA4 gene belong to a GATA family which is consisted of 6 structure-conserved transcription factors. GATA4 gene, which is expressed in cardiac system and endodermal derivatives ${ }^{21}$, is a highly conserved transcriptional factor with seven exons. The GATA4 protein is comprised of TAD, NZF and NLS ${ }^{22}$. Many evidences showed that GATA4 plays significant roles in many stages of heart development, including looping morphogenesis, septation, ventricular myocardium proliferation, and heart contraction ${ }^{1}$. For this reason, GATA4 was considered as regular candidate for CHD genetic screening. Many mutations in GATA4 coding region have been identified as genotype of $\mathrm{CHD}$ patients and not all of them were predicted by bioinformatics tools, such as PROVEAN and SIFT, as pathogenic gene ${ }^{1}$. NLS was considered as crucial role in CHD epidemiology because 5 family cases were reported in this region. Especially in present study, we determined a different protein mutation in the same protein location as reported ${ }^{23}$. In the mouse mutation study, M310V transgenic mice had shown higher incidence of ASD than wild-type control mice ${ }^{24}$, which indicated codon 310 in GATA4 gene is a CHD-related pathogenic coding region. GATA4 p.M310T in this study also proved that and we presume that GATA4 gene may related to tachyarrhythmias too.

$\mathrm{CHD}$ is a heavy load for the young family and whole society. In China, the prevalence is still high although many CHD fetuses were aborted when the prenatal screening predicted highly possible of CHD. In past two decades, cardiac development and genetic studies had provided much detailed information and identified many critical genes in the development of heart. Although many mutants of these genes had been screened out, more biochemical methods and vivo models should be adopted for confirming. A limitation of this study is that only genetic screening methods had been employed but no furthermore study, such as vivo models, 
were used. Further studies will be conducted in the future to study the pathogenic mechanism of CHD family with arrhythmias and the reason for different phenotype with same genotype.

\section{Conclusion}

In this study, we identified a variant in GATA4 gene (c.T929C: p.M310T) in a CHD family with arrhythmias. It suggested that the region coding NLS of GATA4 gene is an important region for development of heart and GATA4 gene may related to tachyarrhythmias.

\section{Abbreviations}

CHD

Congenital heart disease

HTS

high-throughput sequencing

PAVSD

partial atrioventricular septal defect

TFs

transcriptional factors

ASD

atrial septal defect

VSD

ventricular septal defect

AVSD

atrioventricular septal defect

DORV

double outlets of right ventricle

PS

pulmonary stenosis

WES

Whole-exome sequencing

SNVs

Single nucleotide variants

InDels

insertions and deletions

\section{Declarations}

Ethical Approval and Consent to participate: Study approval was obtained from the Committee on Clinical Applications at the Second Xiangya Hospital and the written informed consents were obtained from the patients and patients' parents ( $<16$ years old). 
Consent for publication: The written informed consent for publication of clinical details and/or clinical images was obtained from the parents of participants under the age of 18 and participants themselves (those aged 18 and over).

Availability of data and materials: Yes.

Competing interests: No.

Funding: This work was supported by the National Natural Science Foundation of China (Grant No. 81800290)

Authors' contributions:

Tianli Zhao: study designing and surgical operation;

Guowen sun: data collection;

Yun Zhu: editing the draft;

Yifeng Yang: technique support;

Zhiping Tan: technique support;

Haisong Bu: data collection;

Shijun Hu: manuscript writing and drawing.

Acknowledgements: Thanks for the China Scholarship Council (award to Hu Shijun for 1 year's study abroad at the Munich heart center).

Authors' information: Not applicable.

\section{References}

1. Yu Y, Lei W, Yang J, Wei YC, Zhao ZL, Zhao ZA, Hu S. Functional mutant GATA4 identification and potential application in preimplantation diagnosis of congenital heart diseases. Gene 2018;641:349-354.

2. Zhao QM, Ma XJ, Ge XL, Liu F, Yan WL, Wu L, Ye M, Liang XC, Zhang J, Gao Y, Jia B, Huang GY. Pulse oximetry with clinical assessment to screen for congenital heart disease in neonates in China: a prospective study. Lancet 2014;384(9945):747-54.

3. Lanz J, Brophy JM, Therrien J, Kaouache M, Guo L, Marelli AJ. Stroke in Adults With Congenital Heart Disease: Incidence, Cumulative Risk, and Predictors. Circulation 2015;132(25):2385-94.

4. Wang F, Harel-Sterling L, Cohen S, Liu A, Brophy JM, Paradis G, Marelli AJ. Heart failure risk predictions in adult patients with congenital heart disease: a systematic review. Heart 2019.

5. Pedersen MGB, Olsen MS, Schmidt M, Johnsen SP, Learn C, Laursen HB, Madsen NL. Ischemic Stroke in Adults With Congenital Heart Disease: A Population-Based Cohort Study. J Am Heart Assoc 2019;8(15):e011870. 
6. Bentham J, Bhattacharya S. Genetic mechanisms controlling cardiovascular development. Ann N Y Acad Sci 2008;1123:10-9.

7. Wang J, Cao H, Sun D, Qi Z, Guo C, Peng W, Sun Y, Xie Y, Liu X, Li B, Luo Y, Pan Y, Li Y, Zhang L. Associations between ambient air pollution and mortality from all causes, pneumonia, and congenital heart diseases among children aged under 5 years in Beijing, China: A population-based time series study. Environ Res 2019;176:108531.

8. Nemer M. Genetic insights into normal and abnormal heart development. Cardiovasc Pathol 2008;17(1):48-54.

9. Paige SL, Plonowska K, Xu A, Wu SM. Molecular regulation of cardiomyocyte differentiation. Circ Res 2015;116(2):341-53.

10. Su W, Zhu P, Wang R, Wu Q, Wang M, Zhang X, Mei L, Tang J, Kumar M, Wang X, Su L, Dong N. Congenital heart diseases and their association with the variant distribution features on susceptibility genes. Clin Genet 2017;91(3):349-354.

11. Andersen TA, Troelsen Kde L, Larsen LA. Of mice and men: molecular genetics of congenital heart disease. Cell Mol Life Sci 2014;71(8):1327-52.

12. Guo T, Tan ZP, Chen HM, Zheng DY, Liu L, Huang XG, Chen P, Luo H, Yang YF. An effective combination of whole-exome sequencing and runs of homozygosity for the diagnosis of primary ciliary dyskinesia in consanguineous families. Sci Rep 2017;7(1):7905.

13. Bouma BJ, Mulder BJ. Changing Landscape of Congenital Heart Disease. Circ Res 2017;120(6):908-922.

14. Fahed AC, Gelb BD, Seidman JG, Seidman CE. Genetics of congenital heart disease: the glass half empty. Circ Res 2013;112(4):707-20.

15. Pawlak M, Niescierowicz K, Winata CL. Decoding the Heart through Next Generation Sequencing Approaches. Genes (Basel) 2018;9(6).

16. Yang Y, Muzny DM, Reid JG, Bainbridge MN, Willis A, Ward PA, Braxton A, Beuten J, Xia F, Niu Z, Hardison M, Person R, Bekheirnia MR, Leduc MS, Kirby A, Pham P, Scull J, Wang M, Ding Y, Plon SE, Lupski JR, Beaudet AL, Gibbs RA, Eng CM. Clinical whole-exome sequencing for the diagnosis of mendelian disorders. N Engl J Med 2013;369(16):1502-11.

17. Ng SB, Buckingham KJ, Lee C, Bigham AW, Tabor HK, Dent KM, Huff CD, Shannon PT, Jabs EW, Nickerson DA, Shendure J, Bamshad MJ. Exome sequencing identifies the cause of a mendelian disorder. Nat Genet 2010;42(1):30-5.

18. Bamshad MJ, Ng SB, Bigham AW, Tabor HK, Emond MJ, Nickerson DA, Shendure J. Exome sequencing as a tool for Mendelian disease gene discovery. Nat Rev Genet 2011;12(11):745-55.

19. Williams MR, Perry JC. Arrhythmias and conduction disorders associated with atrial septal defects. J Thorac Dis 2018;10(Suppl 24):S2940-s2944.

20. Ellesoe SG, Johansen MM, Bjerre JV, Hjortdal VE, Brunak S, Larsen LA. Familial Atrial Septal Defect and Sudden Cardiac Death: Identification of a Novel NKX2-5 Mutation and a Review of the Literature. Congenit Heart Dis 2016;11(3):283-90.

21. Patient RK, McGhee JD. The GATA family (vertebrates and invertebrates). Curr Opin Genet Dev 2002;12(4):416-22. 
22. Chen J, Qi B, Zhao J, Liu W, Duan R, Zhang M. A novel mutation of GATA4 (K300T) associated with familial atrial septal defect. Gene 2016;575(2 Pt 2):473-477.

23. Chen Y, Han ZQ, Yan WD, Tang CZ, Xie JY, Chen H, Hu DY. A novel mutation in GATA4 gene associated with dominant inherited familial atrial septal defect. J Thorac Cardiovasc Surg 2010;140(3):684-7.

24. Han H, Chen Y, Liu G, Han Z, Zhao Z, Tang Y. GATA4 transgenic mice as an in vivo model of congenital heart disease. Int J Mol Med 2015;35(6):1545-53.

\section{Figures}

I
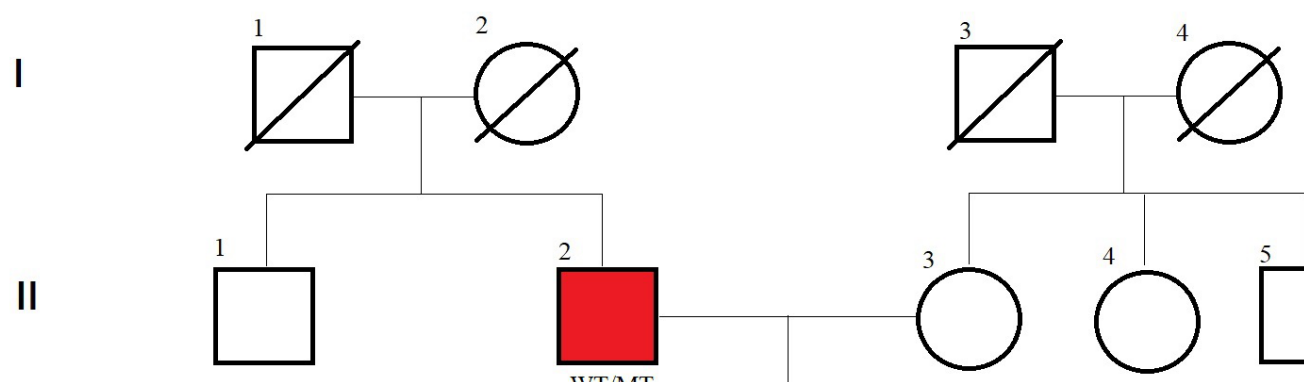

WT:Wild-Type

III

MT: Mutant( c.T929C)
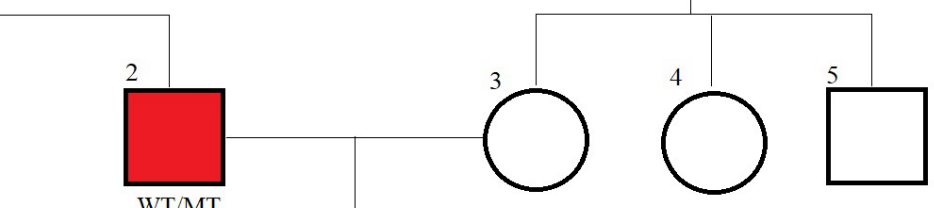

\section{Figure 1}

Pedigree of affected family is comprised of three generations. The squares and circles indicate males and females, respectively. The arrow appoints the proband of the family. The mutation, c.T929C in GATA4, has been demonstrated that segregated in this family. 

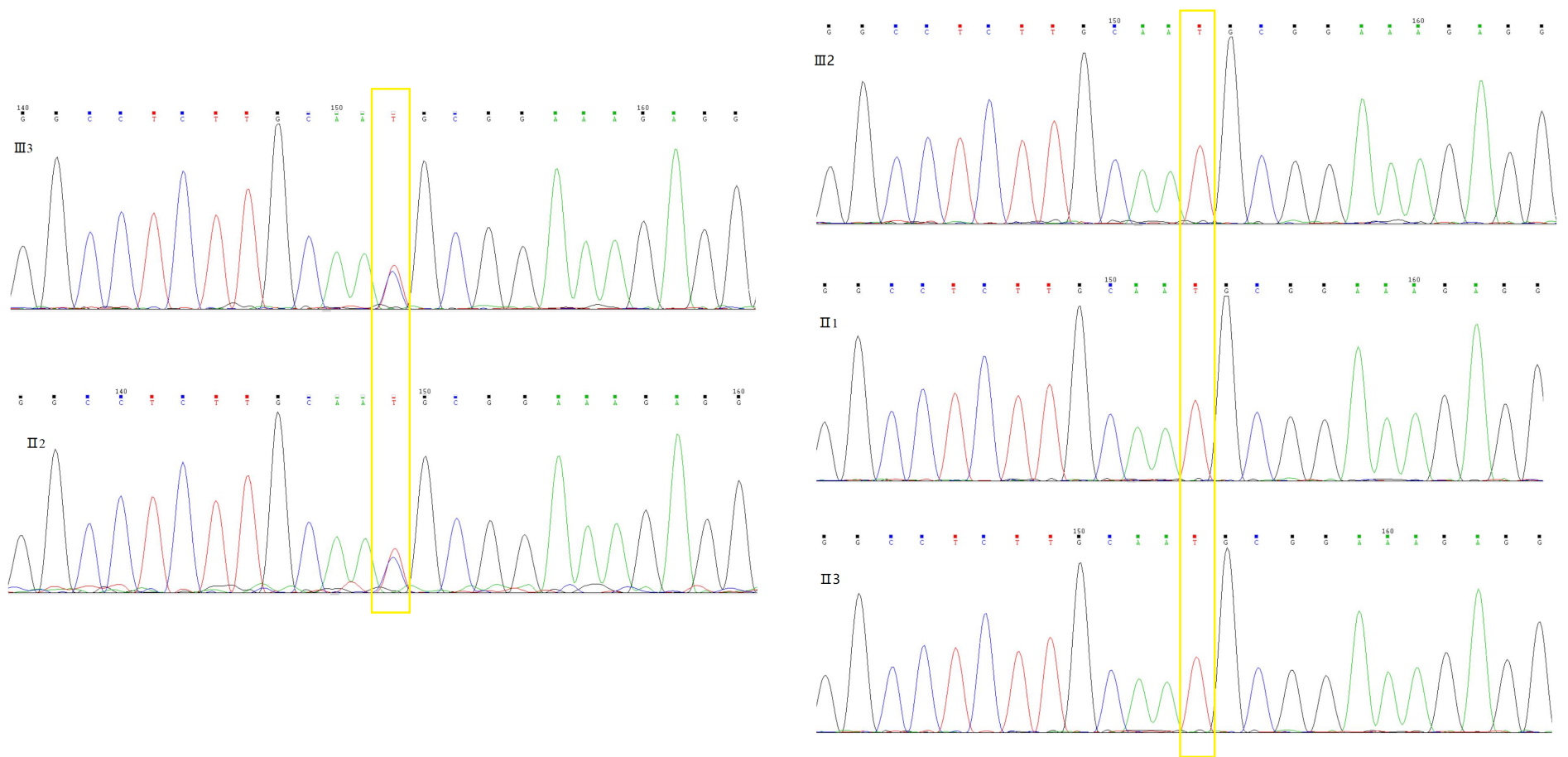

\section{Figure 2}

Sequence chromatogram showing heterozygote state of the nucleotide sequence of GATA4 in c.T929C.
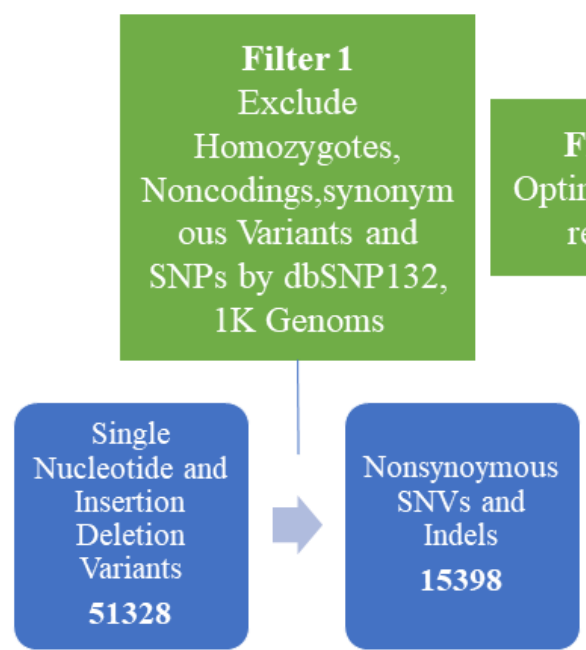

\section{Filter 3}

Filter 2

Opting Exonic

regions
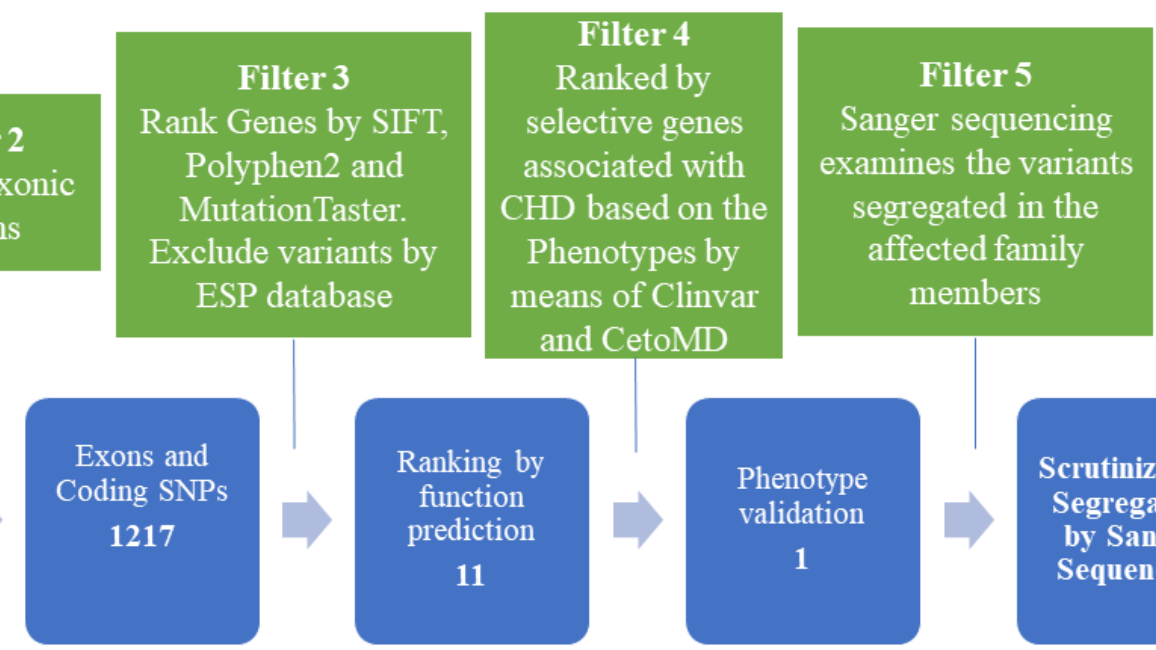

Scrutinize the

Segregation

by Sanger

Sequencing

\section{Figure 3}

Schematic representation of filtering strategies that applied in this research. The filtering process was applied according several strategies which are demonstrated in the schematic representation. 


\section{Supplementary Files}

This is a list of supplementary files associated with this preprint. Click to download.

- tableS142genes.docx

- tableS2.docx 